\title{
DESCRIPCIÓN Y EVALUACIÓN ANATÓMICA DE LESIONES ÓSEAS CRÁNEO-MANDIBULARES EN CETÁCEOS ODONTOCETOS DEL MAR PERUANO
}

\author{
David Montes I. ${ }^{1}$, Alfonso Chavera C. ${ }^{2}$, Marie Van Bresem ${ }^{3}$, Rosa Perales C. ${ }^{2}$, \\ Néstor Falcón P. ${ }^{4}$ y Koen Van Waerebeek ${ }^{3}$
}

\section{Abstract}

\begin{abstract}
A total of 308 skulls and 200 jaws from 20 species and one hybrid of odontocetes cetaceans from the Peruvian Ocean were revised for anatomical description and evaluation of bone lesions. They were grouped into 8 types, plus those at the dental alveoli and osteolitic lesions caused by the nematode Crassicauda sp. From the total bone samples, at least $31.2 \%$ showed one type of craneo-mandibular lesion whereas the bottle nosed dolphin (Tursiops truncatus) was the most affected. Dental alveoli lesions were found in $13.3 \%$ of the samples and bone lesions caused by Crassicauda sp. were in $17.3 \%$ of the skulls. The most frequent lesions were hyperostosis and osteolisis, followed by osteomyelitis, malformations and occipitoatlantoaxial ankylosed joints. Adult males showed higher frequency of bone and dental alveolar lesions and is suggested that the latter type of lesions could be associated with severe cases of osteomyelitis and osteolisis at the maxilar and mandibular level. The pterigoid bone was the most affected by the Crassicauda sp. nematode.
\end{abstract}

Key words: odontocetes, hyperostosis, osteomyelitis, osteolisis, Crassicauda sp.

\section{RESUMIEN}

Se revisaron 308 cráneos y 200 mandíbulas pertenecientes a 20 especies y un híbrido de cetáceos odontocetos del mar peruano a fin de realizar la descripción y evaluación anatómica de lesiones óseas. Se hallaron diversas lesiones óseas, las cuales fueron agrupadas en 8 tipos, además de las halladas a nivel de los alveolos dentarios y de las osteolíticas producidas por el nemátodo Crassicauda sp. El 31.2\% de las muestras presentaron al menos un tipo de lesión cráneo-mandibular, siendo el delfín nariz de botella (Tursiops truncatus) la especie con la mayor frecuencia de lesiones. Las lesiones a nivel de los alveolos dentarios se presentaron en el $13.3 \%$ de la población mientras que las producidas por Crassicauda sp. se observó en el 17.2\% de cráneos revisados. La exostosis y osteolisis fueron las lesiones con mayor frecuencia de presentación, seguido de osteomielitis, malformaciones y anquilosis de la articulación occipitoatlantoaxial. Se observó una mayor frecuencia de lesiones en ejemplares maduros y machos. No se encontró asociación estadística entre la región costera de procedencia y la presentación de lesiones. Las lesiones alveolares dentarias se presentaron con mayor frecuencia en machos adultos, sugiriéndose que estas lesiones podrían asociarse a casos severos de osteomielitis y osteolisis a nivel maxilar y mandibular. Los huesos pterigoides fueron mayormente afectados por el nemátodo Crassicauda sp.

Palabras clave: odontocetos, hiperostosis, osteomielitis, osteolisis, Crassicauda sp.

\footnotetext{
${ }^{1}$ Laboratorio de Anatomía Animal, FMV-UNMSM

${ }^{2}$ Laboratorio de Histo-Patología Animal, FMV-UNMSM

${ }^{3}$ Centro Peruano de Estudios Cetológicos (CEPEC)

${ }^{4}$ Laboratorio de Medicina Veterinaria Preventiva, FMV-UNMSM
} 
En el mar peruano se han registrado 31 diferentes especies de cetáceos, entre ballenas, delfines e incluso una especie de marsopa, lo que equivale a casi el $40 \%$ de las 80 especies de cetáceos conocidas a nivel mundial (García-Godos y Van Waerebeek, 1994). El grupo de los odontocetos, incluidos dentro del orden de los cetáceos, lo conforman animales que poseen dientes en número variado y son homodentos, en lugar de las placas córneas que poseen las ballenas pertenecientes al grupo de los misticetos. Muchas de estas especies son constantemente capturadas, tanto en forma accidental como dirigida, en varias localidades de la costa, lo cual puede traer serias repercusiones en el mantenimiento poblacional (Van Waerebeek et al., 1997). Asimismo, las enfermedades y los traumas se consideran también como factores que pueden afectar el desarrollo óptimo de estas poblaciones (Tarpley, 1987). Neddham (1993) afirma que estas afecciones, además de poder presentarse en delfines en cautiverio o en centros de rehabilitación, afectan a las poblaciones silvestres, haciéndolas más vulnerables para su captura; pudiendo tener, así mismo, cierta relación con los varamientos.

Las lesiones óseas son unas de las afecciones de cetáceos más importantes reportadas en otras latitudes. Mortensen et al. (1992) encontraron cierta relación entre agentes infecciosos y altos niveles de contaminación como causales de la presentación de diversas lesiones óseas en mamíferos marinos. Reyes (1989) halló que los ejemplares correspondientes a poblaciones oceánicas del delfín nariz de botella (Tursiops truncatus), presentaban lesiones en algunos de los huesos craneales ocasionadas por el nemátodo Crassicauda sp.; no ocurriendo así en los ejemplares costeros de la misma especie.

El objetivo de la presente investigación fue la descripción y evaluación anatómica de las lesiones óseas halladas en los cráneos y mandíbulas de cetáceos odontocetos del mar peruano.

\section{MaterRiales y Métodos}

Se revisaron cráneos $(\mathrm{n}=308)$ y mandíbulas $(n=200)$ de 308 esqueletos de 20 especies de cetáceos odontocetos de la costa peruana, incluyéndose un híbrido entre las especies Delphinus sp. y Lagenorhyn-chus obscurus (Cuadro 1). De estas muestras, 300 pertenecen a las colecciones científicas del Centro Peruano de Estudios Cetológicos (CEPEC), ubicado en el distrito de Pucusana (Lima), y a la ONG Áreas Costeras y Recursos Marinos (ACOREMA), con sede en Pisco (Ica). Las otras 8 muestras se obtuvieron en playas de las principales localidades costeras a partir de cadáveres en diversos grados de descomposición.

Las muestras originales de CEPEC y ACOREMA se obtuvieron principalmente a partir de especímenes que eran desembarcados como producto de las faenas de pesca artesanal en los principales puertos y caletas, así como de varamientos. En estos casos, se recolectaban datos de la especie, sexo, longitud estándar y madurez gonadal, entre otros; y la cabeza era seccionada para ser sometida a putrefacción y su posterior limpieza. En los años posteriores a las últimas normas de prohibición, debido a la fuerte vigilancia y supervisión de las autoridades, ya no se produjeron desembarcos de animales enteros, de allí que la mayor parte de las muestras provenían de osamentas halladas en las playas.

Las muestras fueron codificadas, para los fines de análisis del presente estudio, de acuerdo a las tres regiones costeras donde se obtuvieron; las mismas que son definidas por el Ministerio de Pesquería como Perú del Norte [de Puerto Pizarro ( $3^{\circ} 29^{\prime}$ LS) a Puerto Culebras $\left(9^{\circ} 56^{\prime} \mathrm{LS}\right)$ ]; Perú Central [de Huarmey $\left(10^{\circ} 04^{\prime} \mathrm{LS}\right)$ a Laguna Grande $\left(13^{\circ}\right.$ $45^{\prime}$ LS)] y Perú del Sur [de San Juan de

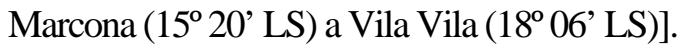

Se diferenció jóvenes y adultos, basándose principalmente en la madurez craneal, debido al escaso número de muestras que con- 
Cuadro 1. Muestras de odontocetos del mar peruano evaluadas en el estudio

\begin{tabular}{|c|c|c|c|c|}
\hline \multirow{2}{*}{ Especie } & \multirow{2}{*}{ Calaveras } & \multirow{2}{*}{ Cráneos } & \multicolumn{2}{|c|}{ Total } \\
\hline & & & $\mathrm{N}^{\mathrm{o}}$ & $\%$ \\
\hline Delphinus capensis & 76 & 33 & 109 & 35.4 \\
\hline Delphinus delphis & - & 2 & 2 & 0.7 \\
\hline Tursiops truncatus & 55 & 16 & 71 & 23.1 \\
\hline Lagenorhynchus obscurus & 31 & 15 & 46 & 14.9 \\
\hline Phocoena spinipinnis & 12 & 26 & 38 & 12.3 \\
\hline Globicephala macrorhynchus & 4 & 8 & 12 & 3.9 \\
\hline Globicephala melas & - & 1 & 1 & 0.3 \\
\hline Stenella attenuata & 2 & 1 & 3 & 1.0 \\
\hline Stenella coeruleoalba & 1 & 1 & 2 & 0.7 \\
\hline Feresa attenuata & 2 & - & 2 & 0.7 \\
\hline Lisodelphis peronii & 1 & - & 1 & 0.3 \\
\hline Peponocephala electra & 3 & - & 3 & 1.0 \\
\hline Steno brevadensis & - & 1 & 1 & 0.3 \\
\hline Pseudorca crassidens & 1 & - & 1 & 0.3 \\
\hline Grampus griseus & 3 & 1 & 4 & 1.3 \\
\hline Kogia simus & 1 & - & 1 & 0.3 \\
\hline Kogia breviceps & 1 & 1 & 2 & 0.7 \\
\hline Mesoplodon peruvianus & 3 & 1 & 4 & 1.3 \\
\hline Mesoplodon grayi & 1 & - & 1 & 0.3 \\
\hline Ziphius cavirostris & 2 & 1 & 3 & 1.0 \\
\hline (Híbrido) & 1 & - & 1 & 0.3 \\
\hline Total & 200 & 108 & 308 & 100.00 \\
\hline
\end{tabular}

taban con la información de la madurez gonadal. Para la determinación de la madurez craneal se usó la metodología aplicada por Van Waerebeek (1993). Sin embargo, este método no fue aplicable para las muestras de la familia Ziphiidae y Physeteridae, así como en las especies del género Globicephala sp. y Pseudorca crassidens debido a que su conformación anatómica difiere del resto de delfínidos.
En las lesiones encontradas en las estructuras óseas cráneo-mandibulares se describió el tamaño, forma, superficie, coloración, consistencia y extensión, siguiendo el orden convencional sugerido por Winter (1968), y se formularon los diversos diagnósticos macroscópicos de las lesiones, los cuales fueron clasificados y agrupados según su similaridad. 
Los grados de afección para las lesiones halladas se definieron como leve, moderado y severo, de acuerdo a la clasificación propuesta por Slauson y Cooper (1990) para lesiones inflamatorias histopatológicas, y adaptándola para las lesiones macroscópicas halladas en la presente investigación.

En los análisis estadísticos se tomaron en cuenta las variables especie, región costera, sexo y madurez craneal o madurez sexual, usándose el programa estadístico SPSS 10.0 y la prueba de chi-cuadrado.

\section{Resultados}

\section{Generalidades}

La madurez craneal se pudo determinar en el 57\% (85/149) de las muestras que pudieron ser revisadas, incrementando de esta manera los datos preexistentes en las colecciones revisadas. Se determinaron ocho (8) tipos principales de lesiones de origen desconocido, clasificadas como disminución de la superficie ósea, hiperostosis, osteomielitis, osteólisis y fístulas, fracturas y/o fisuras, malformaciones, fusión y anquilosis de la articulación occipitoatlantoaxial, y traumas diversos. Además, se encontraron lesiones osteolíticas causadas por Crassicauda sp. (Nematoda, Spiruridae) y otras halladas a nivel de los alveolos dentarios.

El 27.9\% (86/308) de las muestras estudiadas presentó al menos un tipo de lesión a nivel cráneo-mandibular, sin considerar las afecciones alveolares ni las causadas por el nemátode Crassicauda sp. (Cuadro 2). La especie Tursiops truncatus es la que presentó la mayor frecuencia de lesiones, seguido de Delphinus capensis; mientras que Lagenorhynchus obscurus y Phocoena spinipinnis presentaron una menor frecuencia de lesiones; sin embargo, los resultados se expresan en base al número absoluto, sin considerar las diferencias entre número de muestras por especie. Entre las otras especies afectadas se encuentra el Globicephala macrorhyn-chus, Globicephala melas, Grampus griseus, Mesoplodon peruvianus, Feresa attenuata y un ejemplar híbrido, a pesar que se tuvo acceso a un bajo número de muestras. Las demás especies de odontocetos no presentaron ningún tipo de lesión.

La frecuencia de estas lesiones fue mayor en machos (46.8\%) que en hembras (25.9\%, Cuadro 2), así como en ejemplares adultos (42.3\%) sobre jóvenes (16.8\%, Cuadro 3), con base a todas las muestras de cetáceos odontocetos revisadas.

La hiperostosis y las lesiones de tipo osteolíticas se presentaron con mayor frecuencia $(7.5 \%$ y $5.8 \%$, respectivamente), seguidas de la disminución de la superficie ósea (3.9\%), malformaciones (3.6\%) y fracturas y/o fisuras $(2.6 \%)$. La presentación de los diversos tipos de lesiones en las especies de cetáceos odontocetos bajo estudio se encuentra en los cuadros 4 y 5 .

\section{Lesiones}

Disminución de la superficie ósea

Se presentó una pérdida de la superficie ósea en 12 ejemplares de 5 especies, mayormente en hembras y en adultos (cuadros 2 y 3 ). Las lesiones fueron leves y moderadas, a excepción de un caso de severa erosión a nivel del occipital en un ejemplar de Tursiops truncatus.

\section{Hiperostosis}

Este tipo de lesión fue la más frecuente (26.7\%, 23/86), especialmente en adultos (cuadros 2 y 3); siendo Tursiops truncatus la especie con mayor número de ejemplares afectados. Se evidenció un crecimiento de la superficie ósea en forma irregular, siendo el occipital, los frontales, los premaxilares y maxilares los huesos mayormente afectados.

\section{Osteomielitis}

Los huesos afectados con esta lesión mostraron un aumento anormal de tamaño 
Cuadro 2. Número de casos y tipo de lesiones óseas cráneo-mandibulares distribuidas según sexo en odontocetos de la costa peruana

\begin{tabular}{|c|c|c|c|c|c|c|c|c|}
\hline \multirow{3}{*}{ Tipo de lesión } & \multicolumn{8}{|c|}{ Sexo } \\
\hline & \multicolumn{2}{|c|}{$\begin{array}{c}\text { Machos } \\
(\mathrm{n}=79)\end{array}$} & \multicolumn{2}{|c|}{$\begin{array}{c}\text { Hembras } \\
(\mathrm{n}=54)\end{array}$} & \multicolumn{2}{|c|}{$\begin{array}{c}\text { Desconocido } \\
(\mathrm{n}=175)\end{array}$} & \multicolumn{2}{|c|}{$\begin{array}{l}\text { Total } \\
(\mathrm{n}=308)\end{array}$} \\
\hline & $\mathrm{N}^{\mathrm{o}}$ & $\%$ & $\mathrm{~N}^{\circ}$ & $\%$ & $\mathrm{~N}^{\mathrm{o}}$ & $\%$ & $\mathrm{~N}^{\mathrm{o}}$ & $\%$ \\
\hline Disminución de la superficie ósea & 2 & 2.5 & 4 & 7.4 & 6 & 3.4 & 12 & 3.9 \\
\hline Hiperostosis & 9 & 11.4 & 6 & 11.1 & 8 & 4.6 & 23 & 7.5 \\
\hline Osteomielitis & 3 & 3.8 & 1 & 1.9 & 3 & 1.7 & 7 & 2.3 \\
\hline Osteolisis y fístulas & 9 & 11.4 & 0 & 0.0 & 9 & 5.1 & 18 & 5.8 \\
\hline Fracturas y/o fisuras & 4 & 5.1 & 0 & 0.0 & 4 & 2.3 & 8 & 2.6 \\
\hline Malformaciones & 6 & 7.6 & 2 & 3.7 & 3 & 1.7 & 11 & 3.6 \\
\hline Fusión y anquilosis ${ }^{1}$ & 3 & 3.8 & 1 & 1.9 & 1 & 0.6 & 5 & 1.6 \\
\hline Traumas diversos & 1 & 1.3 & 0 & 0.0 & 1 & 0.6 & 2 & 0.6 \\
\hline Total & 37 & 46.8 & 14 & 25.9 & 35 & 20.0 & 86 & 27.9 \\
\hline
\end{tabular}

${ }^{1}$ Fusión v anquilosis de la articulación occipitoatlantoaxial

$\mathrm{n}$ = tamaño poblacional

№ = Número de muestras afectadas con lesiones óseas

Cuadro 3. Número de casos de lesiones óseas cráneo-mandibulares, según tipo y madurez en odontocetos del mar peruano

\begin{tabular}{|c|c|c|c|c|c|c|c|c|}
\hline \multirow{3}{*}{ Tipo de lesión } & \multicolumn{8}{|c|}{ Madurez } \\
\hline & \multicolumn{2}{|c|}{$\begin{array}{c}\text { Jóvenes } \\
\text { n=101 }\end{array}$} & \multicolumn{2}{|c|}{$\begin{array}{c}\text { Adultos } \\
\mathrm{n}=142\end{array}$} & \multicolumn{2}{|c|}{$\begin{array}{c}\text { Sin determinar } \\
n=65\end{array}$} & \multicolumn{2}{|c|}{$\begin{array}{c}\text { Total } \\
\mathrm{n}=308\end{array}$} \\
\hline & $\mathrm{N}^{\mathrm{o}}$ & $\%$ & $\mathrm{~N}^{\circ}$ & $\%$ & $\mathrm{~N}^{\circ}$ & $\%$ & $\mathrm{~N}^{\circ}$ & $\%$ \\
\hline Disminución de la superficie ósea & 3 & 3.0 & 6 & 4.2 & 3 & 4.6 & 12 & 3.9 \\
\hline Hiperostosis & 3 & 3.0 & 16 & 11.3 & 4 & 6.2 & 23 & 7.5 \\
\hline Osteomielitis & 2 & 2.0 & 5 & 3.5 & 0 & 0.0 & 7 & 2.3 \\
\hline Osteolisis y fístulas & 1 & 1.0 & 15 & 10.6 & 2 & 3.1 & 18 & 5.8 \\
\hline Fracturas y/o fisuras & 0 & 0.0 & 8 & 5.6 & 0 & 0.0 & 8 & 2.6 \\
\hline Malformaciones & 5 & 5.0 & 6 & 4.2 & 0 & 0.0 & 11 & 3.6 \\
\hline Fusión y anquilosis & 2 & 2.0 & 3 & 2.1 & 0 & 0.0 & 5 & 1.6 \\
\hline Traumas diversos & 1 & 1.0 & 1 & 0.7 & 0 & 0.0 & 2 & 0.6 \\
\hline Total: & 417 & 16.8 & 60 & 42.3 & 9 & 13.8 & 86 & 27.9 \\
\hline
\end{tabular}

${ }^{1}$ Fusión y anquilosis de la articulación occipitoatlanto

$\mathrm{n}=$ Tamaño poblacional

№ = Número de muestras con lesiones óseas 
Cuadro 4. Número de ejemplares cetáceos odontocetos del mar peruano con lesiones óseas en alvéolos dentarios

\begin{tabular}{|c|c|c|c|c|c|c|c|c|}
\hline \multirow{3}{*}{ Especies } & & & \multicolumn{4}{|c|}{ Madurez } & \multirow{2}{*}{\multicolumn{2}{|c|}{ Total }} \\
\hline & \multicolumn{2}{|c|}{ Joven } & \multicolumn{2}{|c|}{ Adultos } & \multicolumn{2}{|c|}{$\begin{array}{c}\text { Sin } \\
\text { determinar }\end{array}$} & & \\
\hline & $\mathrm{n}$ & $\%$ & $\mathrm{n}$ & $\%$ & $\mathrm{n}$ & $\%$ & $\mathrm{n}$ & $\%$ \\
\hline Delphinus capensis & $0 / 24$ & 0.0 & $16 / 82$ & 19.5 & $0 / 3$ & 0.0 & $16 / 109$ & 14.7 \\
\hline Tursiops truncatus & $5 / 32$ & 15.6 & $8 / 22$ & 36.4 & $2 / 17$ & 11.8 & $15 / 71$ & 21.1 \\
\hline Lagenorhynchus obscurus & $2 / 19$ & 10.5 & $2 / 19$ & 10.5 & $1 / 8$ & 12.5 & $5 / 46$ & 10.9 \\
\hline Phocoena spinipinnis & $0 / 20$ & 0.0 & $1 / 4$ & 25.0 & $0 / 14$ & 0.0 & $1 / 38$ & 2.6 \\
\hline (Otras) & $0 / 6$ & 0.0 & $3 / 15$ & 20 & $1 / 23$ & 4.3 & $4 / 44$ & 9.1 \\
\hline Total & $7 / 101$ & 6.9 & $30 / 142$ & 21.1 & $4 / 65$ & 6.2 & $41 / 308$ & 13.3 \\
\hline
\end{tabular}

Cuadro 5. Casos de lesiones óseas craneales por Crassicauda sp. en cetáceos odontocetos del mar peruano

\begin{tabular}{|c|c|c|c|c|c|c|c|c|}
\hline \multirow{3}{*}{ Especies } & \multicolumn{6}{|c|}{ Madurez } & \multirow{2}{*}{\multicolumn{2}{|c|}{ Total }} \\
\hline & \multicolumn{2}{|c|}{ Joven } & \multicolumn{2}{|c|}{ Adulto } & \multicolumn{2}{|c|}{$\begin{array}{c}\text { Sin } \\
\text { determinar }\end{array}$} & & \\
\hline & $\mathrm{n}$ & $\%$ & $\mathrm{n}$ & $\%$ & $\mathrm{n}$ & $\%$ & $\mathrm{n}$ & $\%$ \\
\hline Delphinus capensis & $5 / 24$ & 20.8 & $26 / 82$ & 31.7 & $0 / 3$ & 0.0 & $31 / 109$ & 28.4 \\
\hline Tursiops truncatus & $5 / 32$ & 15.6 & $6 / 22$ & 27.3 & $5 / 17$ & 29.4 & $16 / 71$ & 22.5 \\
\hline Lagenorhynchus obscurus & $1 / 19$ & 5.3 & $0 / 19$ & 0.0 & $1 / 8$ & 12.5 & $2 / 46$ & 4.3 \\
\hline Grampus griseus & $0 / 2$ & 0.0 & $1 / 2$ & 50.0 & $0 / 0$ & 0.0 & $1 / 4$ & 25.0 \\
\hline Feresa attenuata & $1 / 1$ & 100.0 & $1 / 1$ & 100.0 & $0 / 0$ & 0.0 & $2 / 2$ & 100.0 \\
\hline (Híbrido) & $0 / 0$ & 0.0 & $0 / 0$ & 0.0 & $1 / 1$ & 100.0 & $1 / 1$ & 100.0 \\
\hline (Otras) & $0 / 23$ & 0.0 & $0 / 16$ & 0.0 & $0 / 36$ & 0.0 & $0 / 75$ & 0.0 \\
\hline Total & $12 / 101$ & 11.9 & $34 / 142$ & 23.9 & $7 / 65$ & 10.8 & $53 / 308$ & 17.2 \\
\hline
\end{tabular}

en casi todo su espesor, llegando en algunos casos a producir la disolución del tejido óseo. Se encontró en siete muestras pertenecientes a tres especies afectando los huesos mandibulares, premaxilares y maxilares.
Todos los casos se presentaron en forma leve, con la excepción de un ejemplar de $P h$. spinipinnis (Fig.1), en la que se observó una severa osteomielitis rareficante a nivel mandibular, la cual incluye destrucción del tejido óseo. 


\section{Osteolisis y fístulas}

La rarefacción ósea y formaciones de tractos cavitarios acompañados de disolución del tejido óseo sin regeneración evidente, fueron las características que se observaron en las muestras que presentaron este tipo de lesión; en algunos casos como producto de una notoria osteomielitis. En los casos de fístulas, la destrucción del tejido óseo se produjo formando un trayecto tubular anormal en la mandíbula o el maxilar, desde un alveolo dentario hacia el exterior. La mayor frecuencia se presentó en animales adultos (cuadros 2 y 3) y en la especie Delphinus capensis. Las lesiones fueron en su mayoría moderadas y severas.

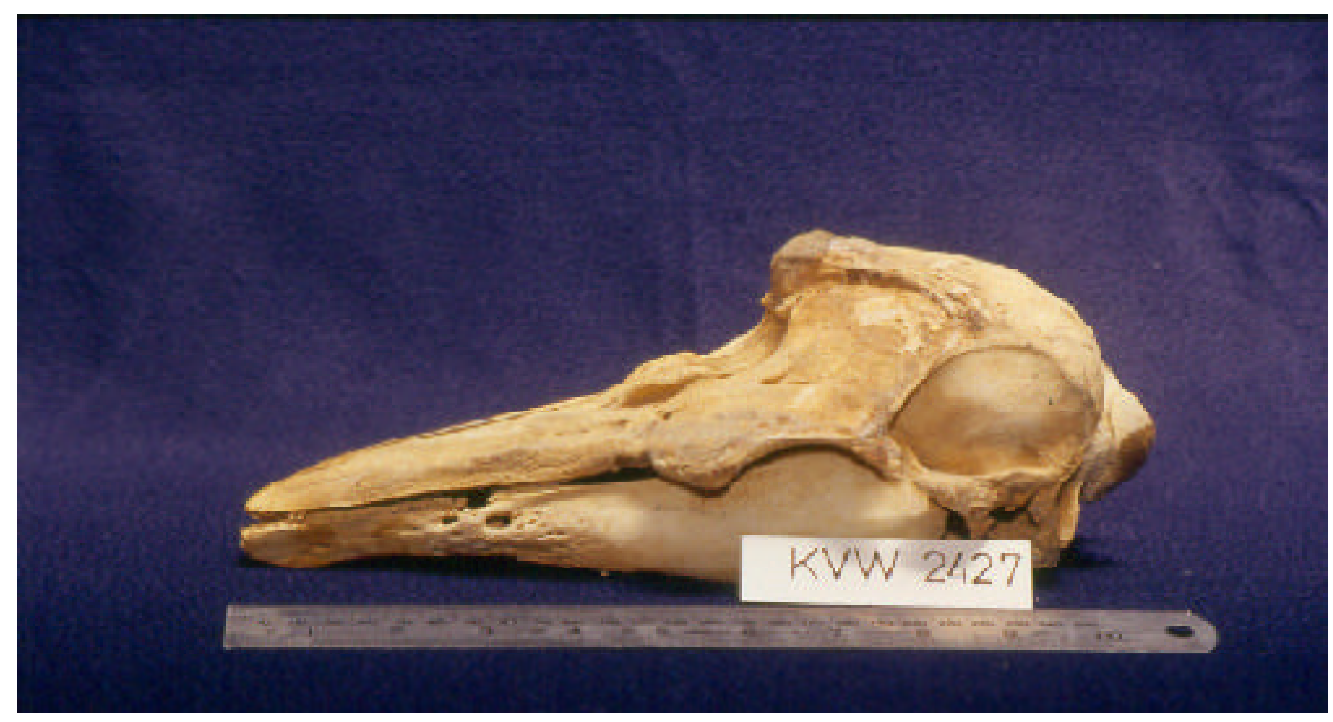

Figura 1. Osteomielitis y osteolisis a nivel de la rama mandibular derecha en un ejemplar de Phocoena spinipinnis

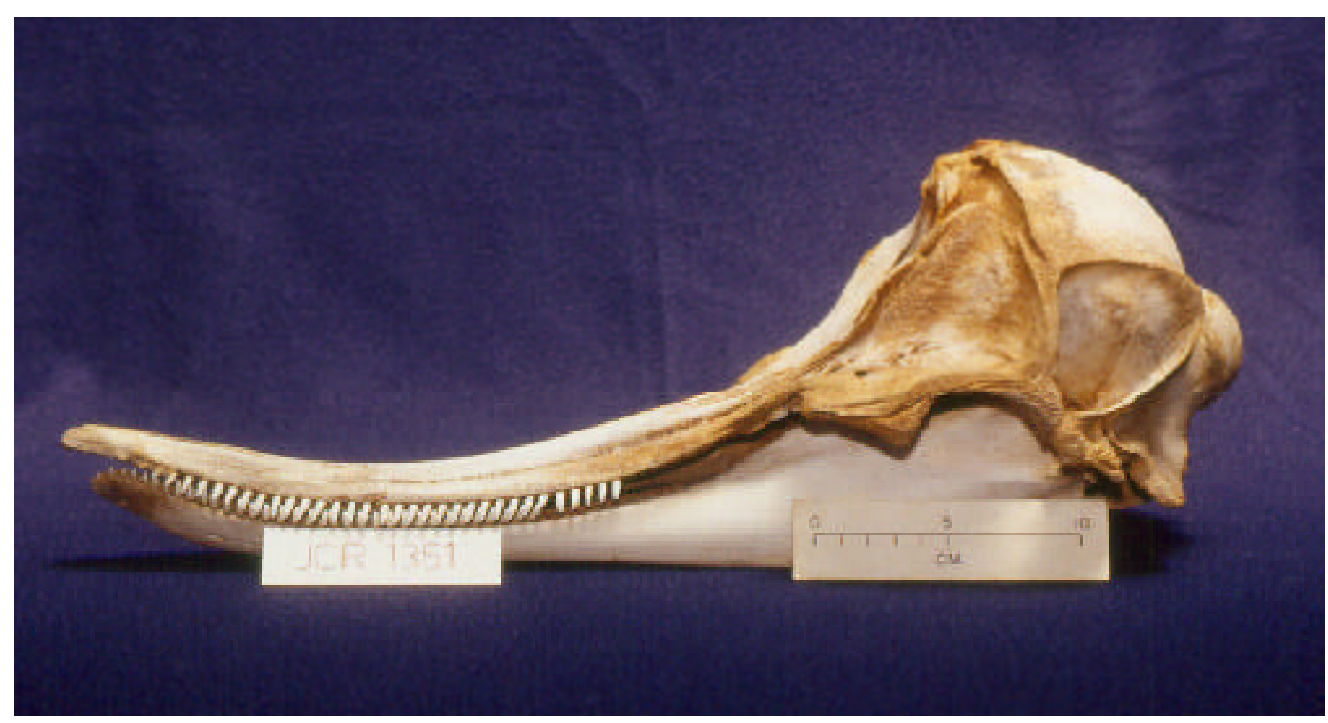

Figura 2. Curvatura dorsal del rostro en un ejemplar de Delpinus capensis 


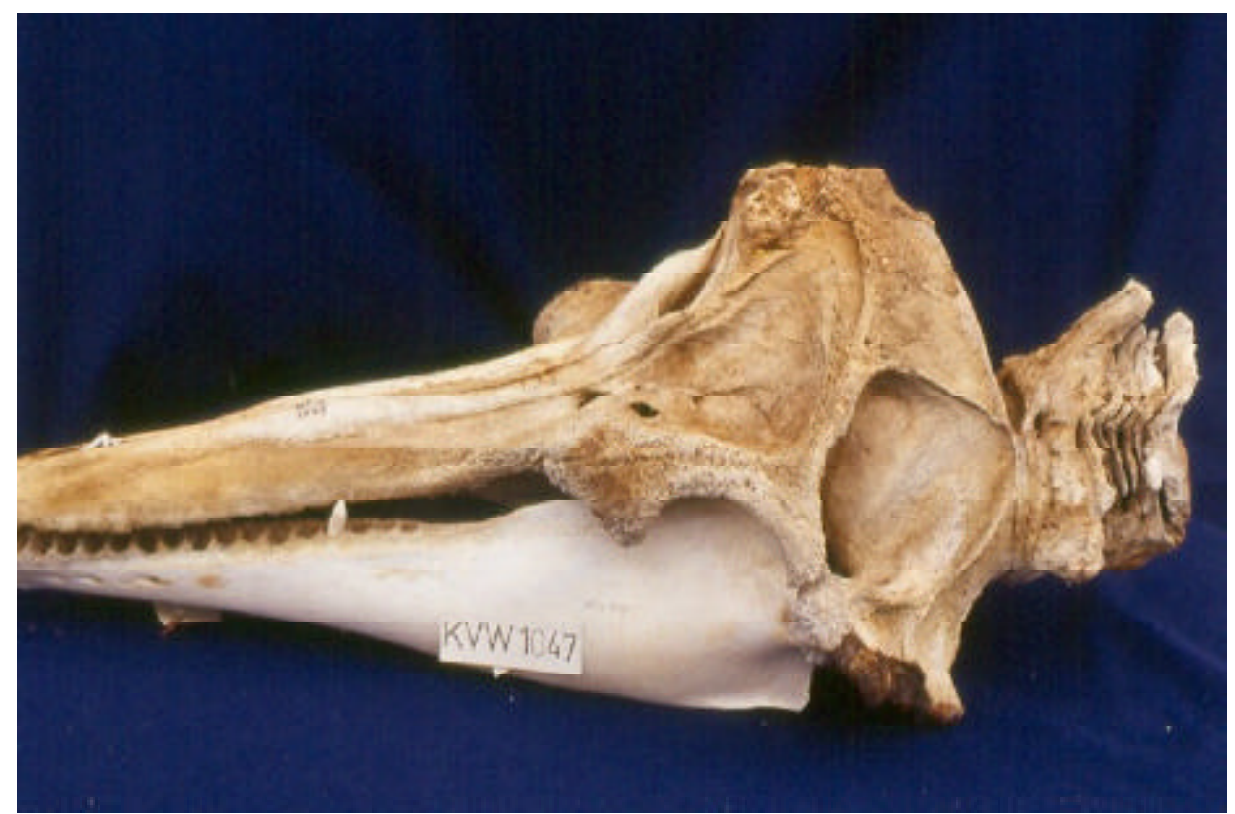

Figura 3. Fusión y anquilosis de la articulación occipitoatlantoaxial en un ejemplar de Tursiops truncatus
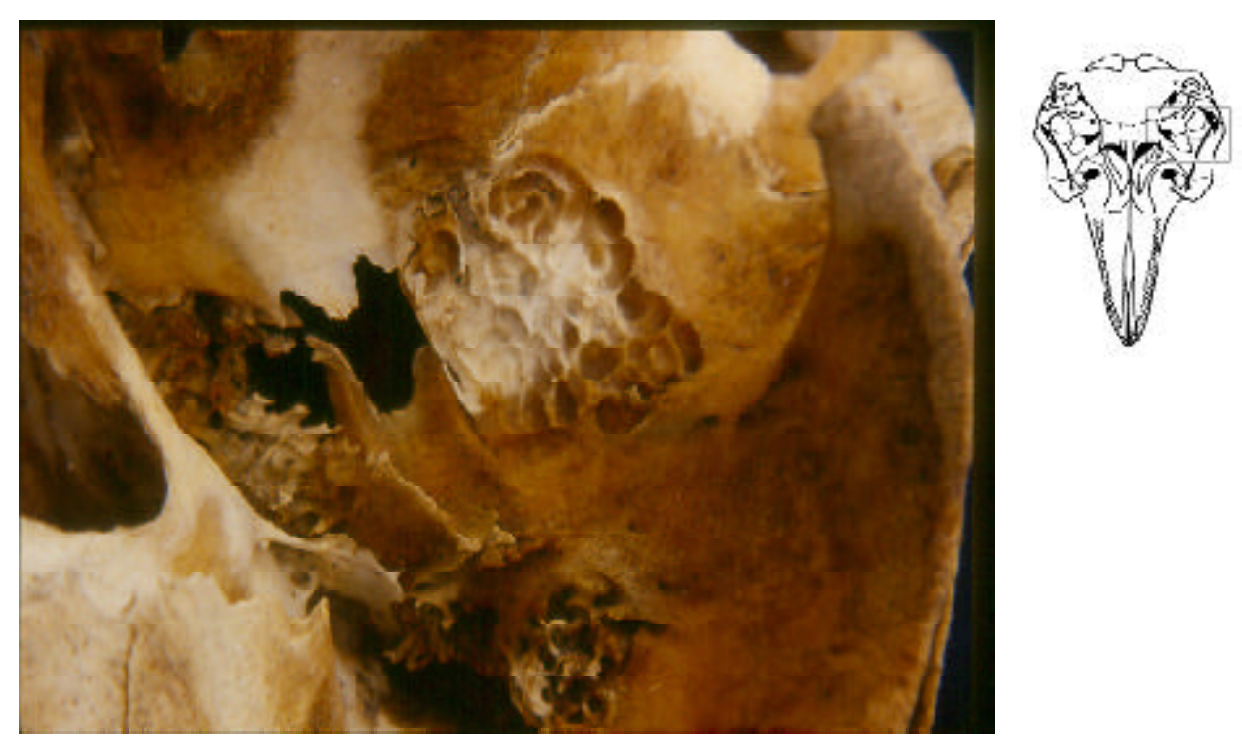

Figura 4. Lesiones osteolíticas características producidas por Crassicauda sp. en un ejemplar de Tursiops truncatus

\section{Fracturas y/o fisuras}

Se notó un engrosamiento de forma lineal sobre la superficie de los huesos afectados, y en algunos casos se formó un "callo óseo". Todos los casos fueron en adultos y a nivel mandibular; con evidencia de una repa- ración ósea completa, a excepción de un ejemplar de Globicephala macrorhynchus, en el cual se observó que la fragmentación de ambas ramas mandibulares no se llegaron a consolidar, a pesar de un evidente proceso inicial de reparación. 


\section{Malformaciones}

Se hallaron 11 ejemplares con este tipo de lesión, pertenecientes a las especies Delphinus capensis, Tursiops truncatus y Lagenorhynchus obscurus, y mayormente en machos. Se encontró braquignatismo y desviaciones de los maxilares (Fig. 2), prognatismo, depresión y protuberancias del occipital y cóndilos articulares.

Fusión y anquilosis de la articulación occipitoatlantoaxial

Se halló en 5 ejemplares de Tursiops truncatus. Se observó la unión anormal del occipital con el atlas y axis, produciendo la inmovilidad de la articulación (Fig. 3). El atlas y las demás vértebras cervicales se encontraban fusionadas, mostrando múltiples estratos óseos en la superficie dorsal y ventral, móviles en algunos casos, con áreas de hiperostosis; evidenciándose una osteomielitis compatible con una espondilitis deformante, así como la elevación de los cuerpos vertebrales, bloqueando parcialmente el agujero magno. Todos los casos se presentaron en forma severa.

\section{Traumas diversos}

Se hallaron 2 muestras con lesiones traumáticas tales como orificios de bordes indefinidos en el occipital, así como de entrada y salida, siguiendo una línea recta con fracturas en su recorrido. Animales de las especies Delphinus capensis y Lagenorhynchus obscurus fueron las afectadas.

\section{Lesiones en alveolos dentarios}

Las afecciones que se presentaron a nivel de los alveolos dentarios fueron casos de obliteración total o parcial, de presencia de tejido óseo de aspecto espongiforme, de lisis de paredes interalveolares, y un caso de inflamación a nivel de los alveolos dentarios.

Se presentaron un total de 41 casos en 7 especies, y con mayor frecuencia en machos y en adultos (Cuadro 4 ).

\section{Lesiones osteolíticas por Crassicauda sp}

Este tipo particular de lesión causada por el nemátodo Crassicauda sp. se presentó en 53 ejemplares (17.2\%) pertenecientes a 6 especies de cetáceos odontocetos.

Se halló en la superficie ventral del cráneo, caracterizándose por la disolución de forma redondeada del tejido óseo, creando en el hueso afectado una superficie con apariencia de pequeños cráteres circulares a manera de cestos o canastas (Fig. 4). En los casos severos, se llegó a evidenciar la perforación del hueso. Se notó una mayor frecuencia de casos con estas lesiones en las muestras provenientes de la región norte $(28.3 \%)$ sobre la región centro (15.0\%). En los huesos afectados, las lesiones se ubicaron con mayor frecuencia en el pterigoides (51.8\%), seguido del frontal y el aliesfenoides; siendo menor en los demás huesos del cráneo.

\section{Discusión}

En base a los resultados obtenidos se puede señalar que la presentación de casos de disminución de la superficie ósea no es exclusividad de animales adultos. A nivel del cráneo, a pesar que la hiperostosis se presentó en forma leve y moderada en casi todos los casos, en los ejemplares de la especie Tursiops truncatus se notó una mayor severidad. Similarmente, De Smet (1977) reporta que los cóndilos del occipital así como la cresta ósea del cráneo formada por el occipital y frontal, eran las estructuras más afectadas en 2 de 12 ejemplares de Tursiops truncatus revisados.

Los casos de osteomielitis fueron leves con excepción de un caso severo que incluyó la destrucción del tejido óseo a nivel mandibular en un ejemplar maduro de Phocoena spinipinnis. Para esta misma especie, Reyes y Van Waerebeek (1995) reportaron casos de osteolisis en el maxilar, premaxilar, nasal y pterigoide. 
Se sugiere que la osteolisis y fistulas pueden tener mayor presentación en animales adultos y puedan ser originadas a partir de infecciones periodontales, que se agravan con el tiempo. En el presente trabajo se observó que los casos de disoluciones del tejido correspondieron principalmente a animales maduros, encontrándose en alveolos dentarios, del maxilar como de la mandíbula. Otros trabajos (Sweeney y Ridgway, 1975) reportan la presencia de dientes afectados con material purulento entre los alveolos, afirmando que esta infección puede cursar con osteomielitis.

La presencia de callos óseos a nivel mandibular fueron observados en la presente investigación, evidenciando la reparación completa de fracturas o fisuras. Otros investigadores también reportaron casos de fracturas con signos de reparación en Tursiops truncatus (Van Bree y Duguy, 1970; De Smet, 1977). Las fracturas pueden relacionarse a causas antropogénicas, así como por peleas de competitividad, dominancia o apareamiento entre ejemplares de una misma especie, o contra depredadores; sin embargo, no existe forma de confirmar o descartar el origen de estas fracturas para los casos hallados en el presente estudio.

Soto y Vega (1997) hacen referencia de una desviación de $6^{\circ}$ hacia la izquierda en la mandíbula de una hembra adulta de ballena picuda de Gray (Mesoplodon grayi) en aguas brasileñas, muy similar a la hallada en el extremo oral de la mandíbula de un ejemplar de Delphinus capensis del presente estudio. También en Brasil, Ott et al. (1996) observaron anomalías craneanas en tres ejemplares adultos de toninas (Pontoporia blainvillei), que incluían curvaciones laterales y dorsoventrales, afirmando que el desarrollo de estas lesiones están asociadas al crecimiento progresivo del animal y que pueden ser relativamente comunes para la especie; sin embargo, en base a los datos existentes, se pro- pone que la presencia de estas alteraciones no están en relación a la edad y que no son comunes. Casos de prognatismo que incluían una laterización de las arcadas dentarias a nivel mandibular fueron observados en las especies Lagenorhynchus obscurus y Tursiops truncatus. No se han hallado reportes similares de esta malformación.

La fusión y anquilosis de la articulacion occipitoatlantoaxial se halló únicamente en Tursiops truncatus, y los reportes de la literatura se refieren solamente a esta especie (Gorzelany et al., 1999; Van Waerebeek et al., 1990) por lo que se sugiere que este tipo de lesión es poco frecuente para cetáceos odontocetos peruanos.

Respecto a las causas, se han reportado luxaciones (Duguy y Van Bree, 1968; Van Bree y Duguy, 1970), traumas relacionados a actividades humanas (enmallamiento o colisión con embarcaciones) (Gorzelany et al.,1999), y agentes infecciosos (Turnbull y Cowan, 1999).

Las características de las lesiones osteolíticas ocasionadas por Crassicauda sp. coinciden ampliamente con las descripciones realizadas por otros autores (Dailey, 1985; Robineau, 1975; Van Waerebeek et al., 1990; Van Waerebeek et al., 1993; Birkun, 2002) en diferentes especies de cetáceos odontocetos.

Las diferencias entre las frecuencias de presentación en las especies estudiadas en este trabajo, sugieren que el hábitat y la dieta podrían tener un papel importante en el ciclo de vida de este nematodo (Van Waerebeek et al., 1993).

El pterigoides fue el hueso más afectado, al igual que en otros estudios (Robineau, 1975; Birkun, 2002); lo que puede ocasionar una interferencia en los sistemas de orientación (Cárdenas et al., 1986). 


\section{ConClusiones}

- Los ejemplares adultos tienen una mayor predisposición a presentar los diversos tipos de lesiones cráneo-mandibulares, a excepción de las malformaciones.

- La hiperostosis fue la lesión de mayor frecuencia de presentación, seguida de la osteolisis.

- Las lesiones en los alveolos dentarios se presentan con mayor predisposición en animales machos y adultos, pudiendo éstas cursar con casos severos de ostemielitis y osteolisis en maxilares y mandíbulas.

- El nemátodo Crassicauda sp. se presenta tanto en animales jóvenes como adultos y en mayor frecuencia en especies de hábitat oceánico como Delphinus capensis; siendo los senos pterigoideos las estructuras mayormente afectadas.

\section{Literatura Citada}

1. Birkun, A. 2002. Natural mortality factors affecting cetaceans in the Black Sea. En: Cetacean of the Mediterranean and Black Seas: State of knowledge and conservation strategies. G. Notarbartolo di Sciara (ed). A report to the ACCOBAMS Secretariat Monaco. Section 16. 13 p.

2. Cárdenas, J.; M. Stutzin; J. Oporto; C. Cabello; D. Torres. 1986. Manual de identificación de cetáceos chilenos. p 23-28. Comité Nacional Pro Defensa de la Flora y Fauna. Santiago, Chile.

3. Dailey, M.D. 1985. Diseases of Mammalia: Cetacea. En: Diseases of Marine Mammals. Vol. IV- 2. O. Kinne (ed). B.A.H. Hamburg. p 805-844.

4. De Smet, W. 1977. The fate of old Bottlenosed dolphins, Tursiops truncatus, in nature as revealed by the condition of their skeletons. Aquat. Mammals 5: 78-86.

5. Duguy, R.; Van Bree. 1968. Catalogue des Cétacés et des Pinnipèdes du Museum d Historie Naturelle de La Rochelle. Annales de la Societé des
Sciences Naturelles de la CharenteMaritime 4: 1-27 (citado por Van Bree, P.; Duguy, R. 1970. Sur queilques aberrations pathologiques chez les petits Cétacés. Der Zoologische Garten Band 39: 1-6.

6. García-Godos, A.; K. Van Waerebeek. 1994. Lista de cetáceos registrados en Perú. VII Congreso Iberoamericano de Biodiversidad y Zoología de Vertebrados. Piura, Perú.

7. Gorzelany, J.; N. Barros; R. De Lynn; J. Litz; L. Wilson. 1999. Bony ankylosis in Atlantic Bottlenose dolphins (Tursiops truncatus) stranded along the Florida Coast. Proceedings $13^{\text {th }}$ Biennial Conference on the Biology of Marine Mammals. Wailea, Hawaii.

8. Mortensen, P.; A. Bergman; A. Bignert; H. Hansen; T. Harkonen; M. Olsonn. 1992. Prevalence of skull lesions in Harbor Seals (Phoca vitulina) in Swedish and Danish Museum Collections: 1835-1988. Ambio 21: 520-524.

9. Needham, D.J. 1993. Cetacean strandings. En: Zoo and Wild Animal Medicine. Current Therapy 3. Fowler, E. (ed). p 415-425. W.B. Saunders. USA.

10. Ott, P.; M. Martins; D. Danilewiz; L. Oliveira; I. Moreno; G. Caon. 1996. Anomalias cranianas em toninhas, Pontoporia blainvillei, no sul do Brasil. Resúmenes de la $7^{\mathrm{a}}$ Reunión de Trabajo de Especialistas en Mamíferos Acuáticos de América del Sur. ${ }^{\text {er }}$ Congreso de la Sociedad Latinoamericana de Especialistas en Mamíferos Acuáticos. Viña del Mar, Chile. 22-25 Octubre.

11. Reyes R.J. 1989. Helmintos parásitos de Tursiops truncatus (Montagu, 1821) (Cetacea : Delphinidae) en aguas de la costa peruana. Tesis de Biólogo. Universidad Ricardo Palma. Lima. 98 p.

12. Reyes, J.C.; K. Van Waerebeek. 1995. Aspects of the biology of Burmeister's Porpoise from Peru. En: Biology of Phocoenids. A. Bjorge; G.P. Donovan (eds). p 349-364. IWC. Cambridge.

13. Robineau, D. 1975. Lésions osseuses liées á la présence de vers parasites du- 
genre Crassicauda (Nematoda, Spiruroi-dea) sur la face ventrale d' un cráne de Tursiops truncatus (Cetacea, Delphini-dae). Annales de la Societé des Sciences Naturelles de la CharenteMaritime. 6: 93-97.

14. Soto, J.; S. Vega. 1997. Primeiro registro da baleia bicuda de Gray, Mesoplodon grayi Haast, 1876 (Cetacea: Ziphiidae) para o Brasil, com referencias osteológicas e a revisão das citações de zifídeos em águas brasileiras. Biociencias, Porto Alegre 5: 69-89.

15. Sweeney, J.; S. Ridgway. 1975. Common diseases of small cetaceans. J. Am. Vet. Med. Assoc. 167: 533-540.

16. Tarpley, R. 1987. Whales and dolphins in veterinary medicine. Southwestern Veterinarian 35: 59-84.

17. Turnbull, B.; D. Cowan. 1999. Synovial joint disease in wild cetaceans. J. Wild. Dis. 35: 511-518.

18. Van Bree, P.; R. Duguy. 1970. Sur queilques aberations pathologiques chez les petits Cétacés. Der Zoologische Garten Band 39: 1-6.
19. Van Waerebeek, K. 1993. Geographic variation and sexual dimorphism in the skull of the dusky dolphin, Lagenorhynchus obscurus (Gray, 1828). Fish Bull. 91: 754-774.

20. Van Waerebeek, K.; J. Reyes; A. Read; J. McKinnon. 1990. Preliminary observations of bottlenose dolphins from the Pacific coast of South America. En: The Bottlenose Dolphin, Chap. 7. S. Leatherwood; R.R. Reeves (eds). p 143154. Academic Press. San Diego.

21. Van Waerebeek, K.; J. Reyes; J. Alfaro. 1993. Helminth parasites and phoronts of dusky dolphins Lagenorhynchus obscurus (Gray, 1828) from Peru. Aquat. Mammals 19: 159-169.

22. Van Waerebeek, K.; M. Van Bressem; F. Felix; J. Alfaro; A. García-Godos; L. Chávez; K. Onton; D. Montes; R. Bello. 1997. Mortality of dolphins and porpoises in coastal fisheries of Peru and southern Ecuador in 1994. Biol. Conservation 81: 43-49.

23. Winter, H. 1968. Guía para la necropsia de los rumiantes domésticos. p 116118. Ed. Acribia. Zaragoza, España. 